\title{
Energy Implications of Production Planning Decisions
}

\author{
Laura Bettoni and Simone Zanoni \\ Department of Mechanical and Industrial Engineering, \\ University of Brescia, Via Branze 38, 25123, Brescia, Italy \\ \{laura.bettoni, zanoni\} @ing.unibs.it
}

\begin{abstract}
The great attention toward environmental issues and especially on energy utilization, leads the European Union to implement the 2009/28/EC Directive, fixing three main goals to be achieved for 2020 by the EU member states: $20 \%$ reduction of the greenhouse gases emission, $20 \%$ increase of using renewable energy and $20 \%$ improving of energy efficiency. To achieve these objectives, new environmental and energy policies must be taken into account not only by governments but also by firms. This work focuses on the energy efficiency effects of production planning decisions in the die casting processes. We present a model based on related literature and on experimental data. The numerical model is based on the relationship between energy consumption of the machines and the production rate of the machines, with the aim to minimize the energy necessary to satisfy the demand of finished products. This model may support manager's decisions so as to minimize energy consumption and consequently environmental impact of die-casting operations.
\end{abstract}

Keywords: Lot sizing, Energy Savings, Die casting, Energy Efficiency.

\section{Introduction}

The increased level of environmental contamination, in particular air pollution, mainly caused by huge usage of non-renewable sources for energy production, the climate changing and the increasing of energy price, led all institutions (public government as well as private companies) all over the world to focus their attention on energy issues. The European Union recently adopted a directive with three main energy objectives: the reduction of $20 \%$ of the greenhouse gases emissions, the increase of $20 \%$ of using renewable energy and the improvement of energy efficiency of $20 \%$, these objectives must be achieved by 2020 . To achieve these objectives, given by European Union through 2009/28/EC Directive, the efforts of all companies with high energy intensive processes are necessary.

For this purpose, the firms firstly have to test their emissions level and their energy efficiency level, furthermore they have to increase the use of renewable energy. Once defined their actual state, they have to analyse the possible corrective actions to apply to improve energy efficiency so as to achieve the European Union's objectives. The energy efficiency, the minimization of energy consumption, the increase of using renewable energy, are now the main objectives for firms, guaranteeing them not only environmental benefits but also consistent economic savings. 
The studies on the energy efficiency assessment are increasing and also the studies on corrective actions to minimize energy consumption. Different approaches are used to decrease energy consumption, the main are the technological and management approaches. There are some studies based on management actions to decrease energy consumption providing scheduling and management techniques and tools to improve energy efficiency in different manufacturing systems. Artigues et al. [1] analysed an industrial case and applied the Cumulative Scheduling Problem (CuSP) to energy issue, naming the specific approach as "Energy Scheduling Problem" (EnSP). Nolde and Morari [9] showed a different electrical energy scheduling in a steel plant: the problem is analysed in order to minimize peak and off-peak energy consumption, which are economically penalized. They used a continuous scheduling model to find a production schedule that achieves the objective of energy cost minimisation. Mouzon et al. [8] analysed the consumption of energy in manufacturing equipment: they observed that applying dispatching rules on the turn-on and turn-off of the machines allows reducing the energy consumption. They use a multi-objective mathematical programming model to decrease energy consumption and total completion time.

Eiamkanachanalai and Banerjee [3] developed a model that jointly determines the optimal run length and the rate of production for a single item; the output rate is the decision variable and the production cost per unit is defined as a quadratic function of the production rate; furthermore the desirability or undesirability of unused capacity, a linear function, is integrated in the model. Christoph H. Glock [4], analysed the total cost of a production system composed by single item worked by single facility and delivered to the subsequent stage in batch shipment, where the production rate is variable. He studied the case of equal- and unequal- sized batch shipments and proposed a solution procedure for the models. The numerical study shows that the variable production rate may reduce inventory carrying cost and thus the total cost of the system. Glock [5] analysed also the batch sizing with variable controllable production rates in a multi stage production system, reaching to the same conclusion.

The main aim of this research is to find possible actions to minimize energy consumption and costs looking at the energy consumption of the production machines in their different operative states. During the idle state the machine is on but it doesn't produces, however, all the auxiliary components are turned on and consume a lot of energy; while during the production state the machine is on and may produces at different production rates. Producing at different production rates implies that the consumption of energy depends on the production rate with which the machine is running. Therefore, to minimize energy consumption it is necessary to put the attention on the idle time of the process, where the machine consumes energy but doesn't produce anything, and on the production rate of the machine, that influences the specific energy consumption. We have proposed an analytical model based on the relationship among energy consumption, idle time, production rate and the demand rate, with the aim to find a trade-off among the variables that minimizes the energy and total costs. We also present a numerical analysis to show the applicability of the model in the field of die casting.

This paper is structured as follow: in section 2 the system studied, with reference to the die casting process, is described, in the section 3 the model developed is presented. The numerical results are offered in section 4 and in section 5 main conclusions with some further development are reported. 


\section{System Description}

This study particularly looks at die casting process, with reference to the cold chamber configuration suitable for aluminium processing [2], where there is high energy consumption, both electric and thermal. In particular the use of furnace for melting metal and holding molten metal at a given temperature for a time period, coupled with the die casting machine, have made this area an important case study for the reduction of energy consumption using production management levers. The furnace uses both electrical (expressed in $[\mathrm{kWh}]$ ) and thermal energy (expressed in $\mathrm{m}^{3}$ of natural gas), while the die casting machine uses only electrical energy (expressed in $[\mathrm{kWh}]$ ). So as to be able to compare the different energy sources we have used as main energy unit the [TOE] (tonne of oil equivalent).

The process consists of three main phases: the melting and the holding phases, belonging to the heating operation, and the press phase. In the melting phase the ingots are introduced in the furnace to be melted; the energy used in this phase is very high due to the high temperature needed to melt the metal, the energy supplied is electrical and thermal. The holding phase is needed to maintain the metal in molten state during the non-production time, i.e. the idle state. During the press phase, the molten metal is forced into a mould cavity under high pressure: the energy supplied is only electrical.

The system uses different levels of power in the different phases: during the melting the furnace to achieve high temperature to melt the metal consumes high thermal power, while during the holding the furnace consumes only the power to maintain the metal in molten state, i.e. the idle power. The die casting machine consumes only during production (processing and idle power), in fact it is turned-off at the end of the shift of production.

Our objective is to find the trade-off between the energy consumption of the production state and the energy consumption of the idle state, with the aim to increase energy efficiency and decrease energy cost.

\section{Model}

The model is based on the comparison between the consumption of energy during different states of the two machines of the system, assuming a variable production rate. The sum of Idle Energy Consumption (IEC) and Production Energy Consumption (PEC) is the Total Energy Consumption (TEC).

Once fixed the cycle time $T$ of the process, varying the production rate, the idle time and the production time to satisfy the demand changes. In particular increasing the production rate the production time decreases and the idle time increases. The specific energy consumption (SEC) during production state decreases while increasing the production rate (Gutowski et al. [6]). The SEC is defined as the amount of energy required for processing a specific amount of product (i.e. in the die-casting expressed in $\mathrm{kg}$ of die-casted aluminium) and can be expressed in [TOE/kg].

Moreover the energy required for processing the material is inversely related to the production rate $p$, of the equipment adopted. This relationship is mainly due to the presence of the idle power required by the process, which is constant even if the production rate adopted may vary, as observed by Gutowski et al. [7]. 
Based on experimental data in the die casting process the relationship between energy required for processing one kilogram of aluminium (named $e$ ) and $p$ can be expressed in the following way:

$$
e_{p}=\alpha * p^{-\beta}
$$

where $\alpha$ and $\beta$ are the coefficients that depends on the specific die casting equipment adopted, and $p$ is the production rate implemented.

Let us introduce the following notations:

- $\quad E_{\text {idle }}$ energy required during idle time of the process, expressed in [TOE];

- $\quad W_{0}$ the idle power of the processing equipment, expressed in [TOE/h];

- $\quad t_{i}$ the idle time varying with production rate, expressed in [h];

- $\quad t_{p}$ the production time, expressed in [h];

- $\quad T$ the cycle time, expressed in [h];

- $\quad e_{p}$ the specific energy consumption, expressed in [TOE/kg];

- $\quad E_{\text {prod }}$ energy required during production time, expressed in [TOE];

- $\quad D$ the demand, during the cycle time, expressed in $[\mathrm{kg}]$;

The Total Energy Consumption required for processing aluminium in both stages (furnaces and die casting machine), during idle time (i.e. $E_{\text {idle, }}$ that is the energy required for holding the molten aluminium in the furnace) and during the production (energy required for melting the aluminium ingots and running the die casting machine) can be evaluated as following reported:

$$
\operatorname{TEC}(p)=E_{\text {idle }}+E_{\text {prod }}=W_{0} \cdot t_{i}+e_{p} \cdot \mathrm{D}=W_{0} \cdot t_{i}+\alpha \cdot \mathrm{p}^{-\beta} \cdot \mathrm{D} .
$$

It should be considered that the demand of products during the cycle time is considered as constant and must be satisfied with no stock out or backorder.

The TEC is a function of idle time and production time, the former one depends on the production rate, so as to completely satisfy the given demand according to the following relationship:

$$
t_{i}=T-t_{p}=T-\frac{D}{p}
$$

\section{$4 \quad$ Case Study}

So as to show the applicability of the model proposed, in this section we will present a numerical analysis based on real data collected from a die casting firm. Based on real data historically collected it has been possible to obtain the relationship between the specific energy consumption [expressed in TOE $/ \mathrm{kg}$ ] and the production rate, reported in Fig. 1. 


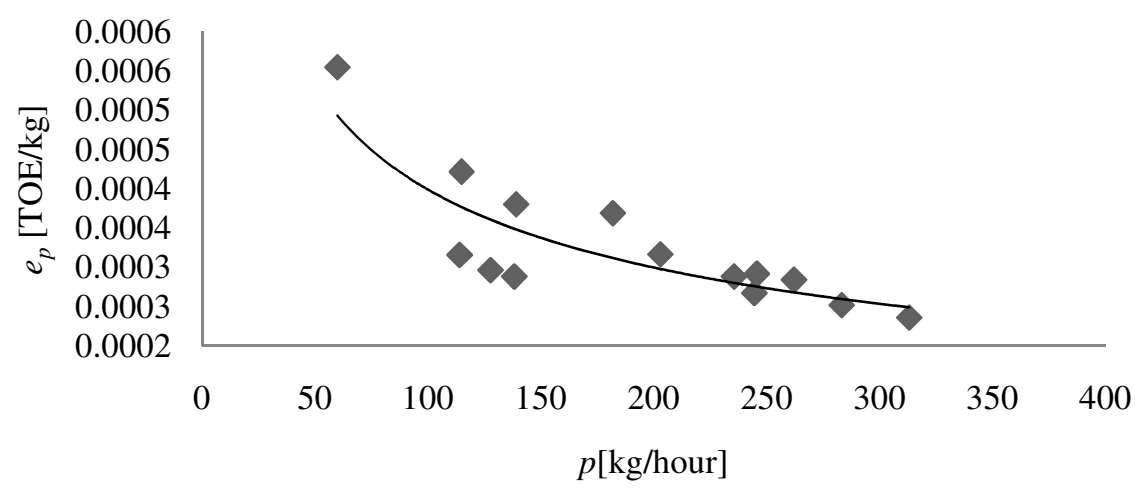

Fig. 1. Specific Energy Consumption for different production rate

In this case the relationship can be expressed with the following equation:

$$
e_{p}=0.0027 * p^{-0.412}
$$

Moreover we have considered the following two assumptions:

1. the cycle time $T$ consist of 24 hours.

2. the maximum production rate of the equipment is $p=500[\mathrm{~kg} / \mathrm{hours}]$.

We have analysed the trend of TEC varying the production time available for production and the corresponding idle time necessary for the holding phase. The production time was varied between 24 hours of production and the minimum hours necessary to satisfy the demand, i.e. if the demand is 2000 [kg/day] and the maximum production rate is $500[\mathrm{~kg} / \mathrm{h}]$ the minimum production time considered was 4 hours. Then, the corresponding idle time considered varied in the range between zero, i.e. 24 hours of production, and the maximum idle time given the constraint on the maximum production rate, i.e. 20 hours of idle time.

This analysis has been performed for two different value of demand: in the first case the demand value is set to 3500 [kg/day], in the second case is set to 7000 [kg/day].

The demand should be considered distributed throughout the week; therefore it is not considered the option of producing, without stops, during several days and then switch off the furnace for the rest of the days.

\section{CASE 1: $\mathrm{D}=3500[\mathrm{~kg} / \mathrm{day}]$}

In Figure 2 we can see the trend of the Total Energy Consumption and its two components: the Idle and Production Energy Consumption. Varying the idle time between zero, i.e. all the cycle time is occupied by the production and the holding phase doesn't exist, and the maximum idle time possible, the PEC decrease, while the IEC increase. We have the minimum TEC when the idle time is zero, so it is better to work on a 3 shifts arrangement. 


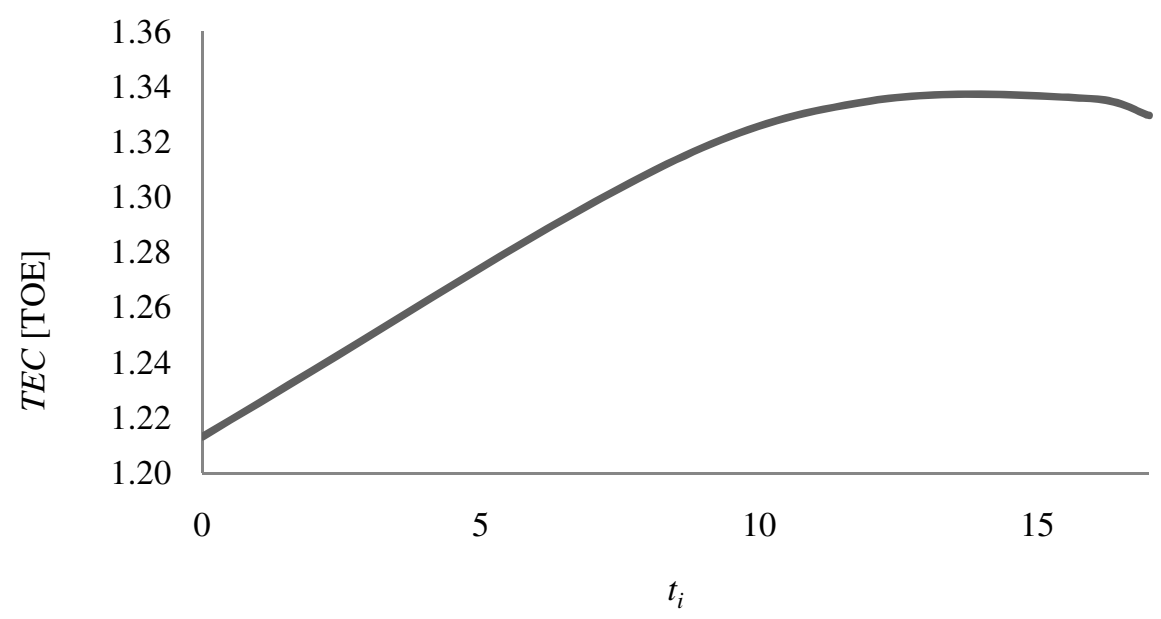

Fig. 2. Trend of TEC for $D=3500$ for different values of idle time

\section{CASE 2: $\mathrm{D}=7000[\mathrm{~kg} /$ dayl}

When the demand increase the trend of TEC changes and the minimum TEC occurs at the maximum idle time and minimum production time possible, which in this case are $[t i ; t p]=[12.8 ; 11.2]$. The analysis suggests that it is better, when the demand increases, to increase the production rate and to work only for two shifts, leaving the furnace in holding phase for the remaining time.

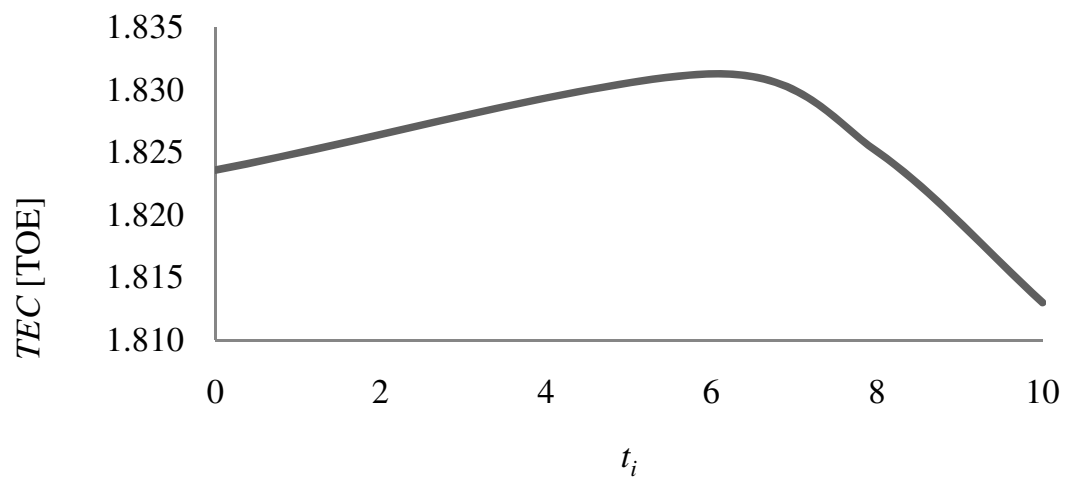

Fig. 3. Trend of TEC for $D=7000$ for different values of idle time

If we analyze the trend of the Specific Energy Consumption in $[\mathrm{kWh} / \mathrm{kg}]$ while varying the demand rate, it is possible to determine the optimal production planning arrangement, in terms of idle time for each production day, in order to minimize the Total Energy Consumption. 


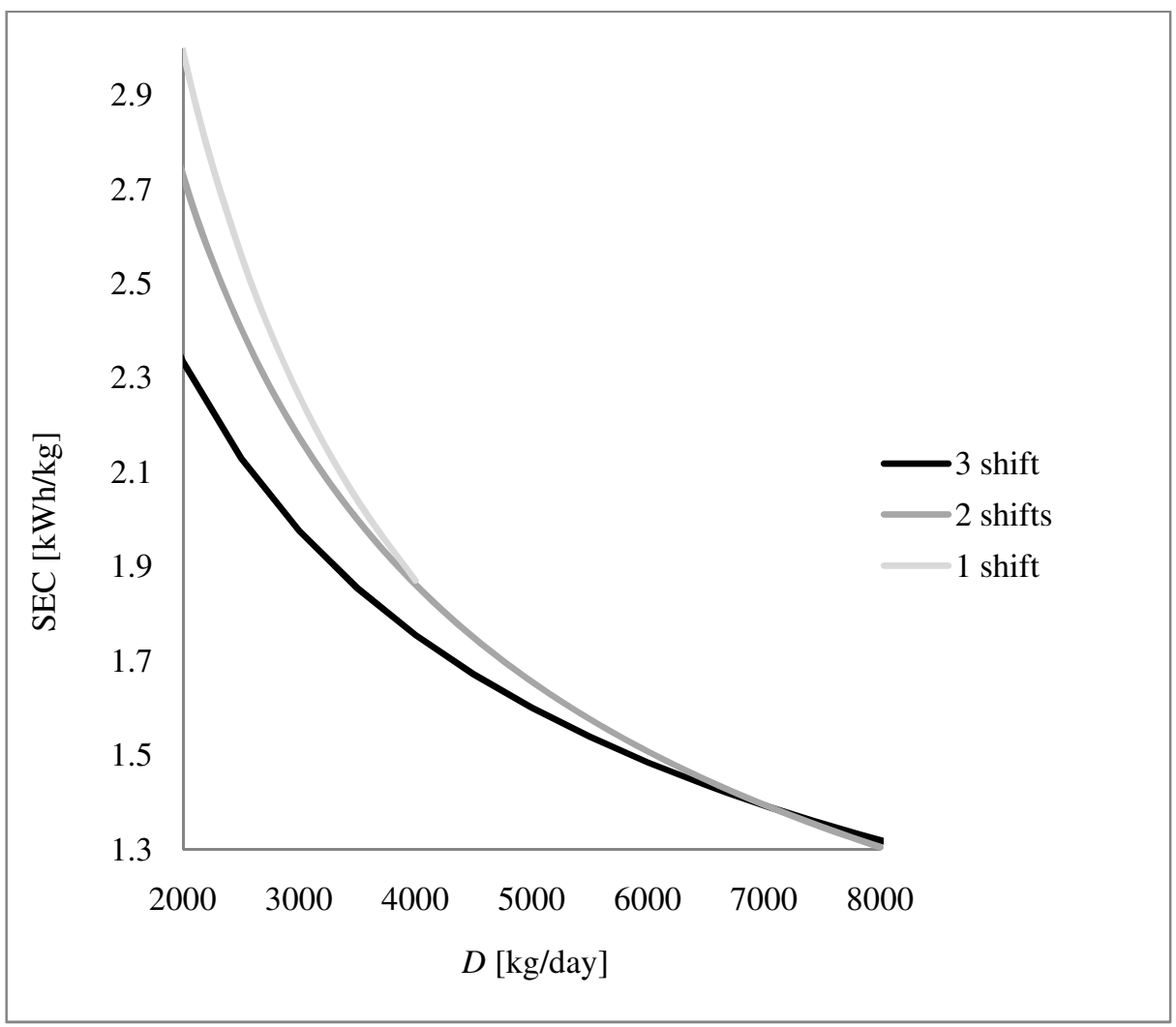

Fig. 4. Trend of SEC

Figure 4 shows the SEC increasing the demand between 2000 and 8000 [kg/day], for three different production planning arrangements: three shifts a day, two shifts a day and only one shift a day, considering 8 production hours for each available shift. As expected, the specific energy consumption decreases while increasing the demand, the minimum value (in our study about $1 \mathrm{kWh} / \mathrm{kg}$ ), corresponds to the variable energy consumption, while for lower demand the SEC is greater due to energy fixed costs (Gutowski, 2006).

The graph can be divided in two areas, as shown in Figure 5, for demand rate less than $7000 \mathrm{~kg} /$ day it is better to plan the production on 3 shift basis, while increasing the demand it is better to plan the production on 2 shifts. This better explain what we have shown in figure 2 and 3.

This effect can be seen as an energy paradox: when the demand increases over a certain limit, instead of arranging a production plan according to 3 shifts, so as to minimize the TEC it is better to increase the production rate of the machines, in order to satisfy the demand using only two production shifts and then leave the machines in idle state for the other shift. 


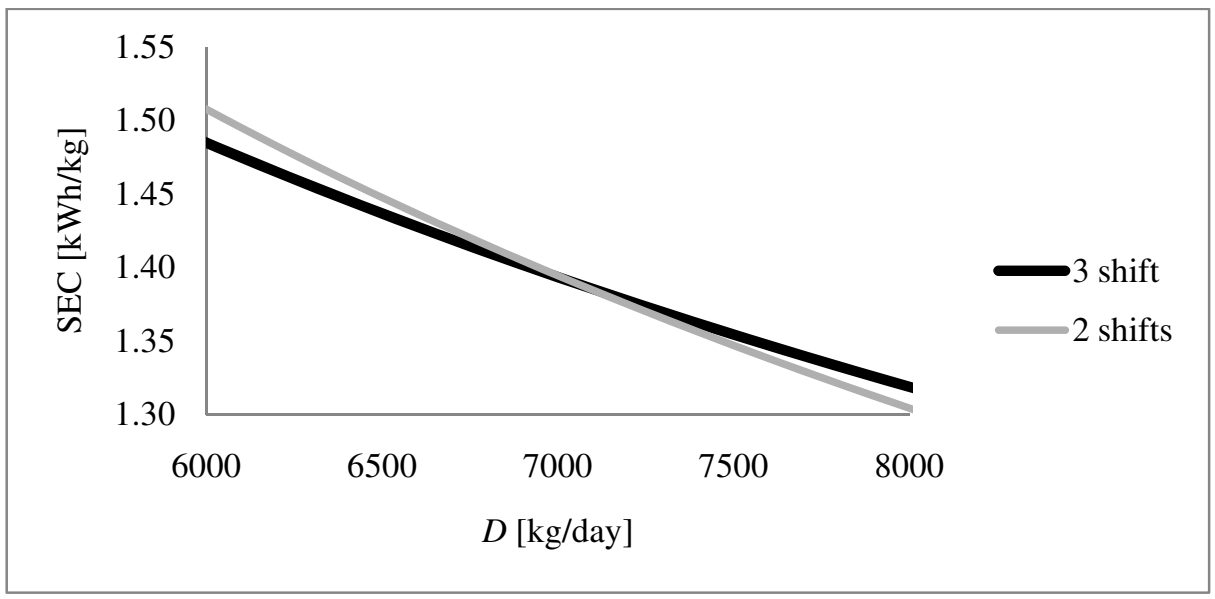

Fig. 5. Detail of the trend of SEC

This can be explained because increasing the production rate the specific energy consumption decreases significantly (e.g figure 1) and this decrease, over a certain limit of the daily demand, can compensate the increase of energy consumption caused by the holding phase during the idle time.

\section{Conclusions}

Aim of this study has been to look at production planning levers so as to reduce energy consumption and consequently energy costs in die casting processes. We have developed an analytical model to express the relationship between Production Energy Consumption, Idle Energy Consumption, and the production rates of the machines so as to find the minimum Energy Consumption and consequently the minimum Total Cost of the system satisfying the given demand in the time window considered.

The numerical analysis for different cases, based on the different demand rate settings, revealed the optimal planning policy to adopt (i.e. the optimal production rate and idle time, and consequently the shifts arrangement), so as to minimize energy consumption.

Further developments can be to extend the model and the numerical study to different production systems, such as metal forging or metal forging processes, where furnaces for warming and holding materials are key elements of the processes.

\section{References}

1. Artigues, C., Lopez, P., Hait, A.: The energy scheduling problem: Industrial case-study and constraint propagation techniques. International Journal Production Economics (2011), doi:10.1016/j.ijpe.2010.09.030

2. Boothroyd, G., Dewhurst, P., Knight, W.: Product design for manufacturing and assembly. Marcel Dekker, New York (2002) 
3. Eiamkanchanalai, S., Banerjee, A.: Production lot sizing with variable production rate and explicit idle capacity cost. International Journal Production Economics 59, 251-259 (1999)

4. Glock, C.H.: Batch sizing with controllable production rates. International Journal of Production Research 48(20), 5925-5942 (2010)

5. Glock, C.H.: Batch sizing with controllable production rates in a multi - stage production system. International Journal of Production Research, 1-23, iFirst (2011)

6. Gutowski, T., Dalquist, S.: Life cycle analysis of conventional manufacturing techniques: die casting. Working draft (2004)

7. Gutowski, T., Dhamus, J., Thiriez, A.: Electrical energy requirements for manufacturing processes. In: 13th CIRP International Conference on Life Cycle Engineering (2006)

8. Mouzon, G., Yildirim, M.B., Twomey, J.: Operational methods for minimization of energy consumption of manufacturing equipment. International Journal of Production Research 45(18), 4247-4271 (2007)

9. Nolde, K., Morari, M.: Electrical load tracking scheduling of a steel plant. Computers and Chemical Engineering 34, 1899-1903 (2010) 